\title{
NÍVEIS SÉRICOS DE HEMOGLOBINA GLICADA EM UMA POPULAÇÃO ATENDIDA EM UMA ESTRATÉGIA SAÚDE DA FAMÍLIA, NA CIDADE DE CRICIÚMA (SC)
}

Helena Ferreira Demetrio Acadêmica do Curso de Medicina, Universidade do Extremo Sul Catarinense, helena_demetrio@hotmail.com Joana Barata Gomes Acadêmica do Curso de Medicina, Universidade do Extremo Sul Catarinense, jo_barata@hotmail.com Kristian Madeira Doutor, Universidade do Extremo Sul Catarinense, kristianmadeira@gmail.com Ana Lucia Camargo Fagundes Mestra, Universidade do Extremo Sul Catarinense, analuciacf@terra.com.br

Celso Carneiro Carvalho

Acadêmica do Curso de Medicina, Universidade do Extremo Sul Catarinense, celsocarneiro_7@hotmail.com

\section{RESUMO}

O Diabetes Mellitus tipo 2 (DM2) é um conjunto heterogêneo de distúrbios metabólicos. É uma doença crônica e silenciosa que ocorre por uma deficiência relativa de insulina, ocasionada por aumento da resistência periférica, gerando um estado de hiperglicemia que, em longo prazo, pode levar a lesóes 
microvasculares, macrovasculares e neuropáticas. Idade mais avançada, atividade física reduzida e, principalmente, a obesidade de longa duração promovem a expressão da doença em indivíduos com susceptibilidade genética. $O$ perímetro da cintura e o perfil lipídico podem ser considerados como fatores de risco. Frente a isso, esta pesquisa procurou verificar os níveis séricos de hemoglobina glicada (A1c) em uma população atendida em uma Estratégia Saúde da Família, na cidade de Criciúma (SC), relacionando a mesma com a idade, gênero e perfil lipídico. Realizou-se um estudo observacional, retrospectivo e de abordagem quantitativa, por meio da análise dos prontuários de indivíduos que frequentaram a Estratégia Saúde da Família, do Bairro Primeira Linha, na cidade de Criciúma (SC), no ano de 2014. Os dados foram analisados por meio do software IBM Stastistical Package for the Social Sciencies (SPSS), versão 22.0. Adotou-se um intervalo de confiança de $95 \%$ e um nível de significância $\alpha=0,05$. A amostra foi composta inicialmente por 128 prontuários, sendo que 86 apresentaram os níveis séricos de hemoglobina glicada (A1c). Os indivíduos eram predominantemente do sexo feminino $(68,6 \%)$, com média de idade de 49,16 anos $( \pm 16,83)$. A taxa de prevalência pontual de DM2 foi estimada em 23,3\%. A amostra demonstrou que a população com menos de 60 anos foi mais suscetível ao desenvolvimento de DM2 (pré-diabetes), enquanto a terceira idade possuiu maior porcentagem de diagnóstico $(55 \%)$ ( $\mathrm{p}=0,001)$. Verificou-se que a população com idade inferior a 60 anos possuiu maior porcentagem da condição clínica de pré-diabetes, encaixando-se, modernamente, sob o título de categoria de risco para diabetes. $\mathrm{O}$ DM2 pode ser prevenido, os pacientes pré-diabéticos devem alterar seu estilo de vida, realizar modificação de hábitos alimentares, redução de peso e aumento da atividade física.

Palavras-chave: Diabete Mellitus tipo 2; Estado Pré-Diabético; Perfil de Saúde. 